\title{
Del aula al laboratorio, tres mujeres científicas en la historia de la psicología argentina
}

\section{Da sala de aula ao laboratório, três mulheres cientistas na história da psicologia argentina}

\section{From the classroom to the laboratory, three women scientists in the history of Argentinian Psychology}

\author{
María Andrea Piñeda* \\ Universidad Nacional de San Luis - UNSL, San Luis, Argentina
}

\begin{abstract}
RESUMEN
Se describe la integración de tres mujeres argentinas a la carrera científica durante la década de 1960 desde sus estudios doctorales en un laboratorio extranjero. Se analizan los auspicios personales y los apoyos en políticas públicas que les otorgaron oportunidades para producir y gestionar ciencia. Se trata de Hermelinda Fogliatto, Rosalía Paiva y Ana María Insúa quienes comenzaron su actividad profesional en el ámbito educativo accediendo a la Psicología. Cuando en el país no existía tradición de promover doctorados en esa disciplina, ellas lo lograron en el Laboratorio de Psicometría de la Universidad de Loyola (Chicago) bajo la dirección de Horacio Rimoldi, quien las impulsó en su carrera. Desde modelos factoriales, condujeron investigaciones sobre procesos de pensamiento para la resolución de problemas que se convirtieron en referencia internacional. Las tres mujeres se desempeñaron en instituciones de Estados Unidos con financiamiento de agencias oficiales de salud y educación. Dos de ellas regresaron a Argentina donde ingresaron al Consejo Nacional de Investigaciones Científicas y Técnicas. Se discute el rol de dicha institución en la promoción de la investigación en psicología, el lugar de la misma en las carreras universitarias y su incidencia en el perfil de psicólogo.
\end{abstract}

Palabras clave: Historia de la psicología, mujeres, carrera científica, procesos de pensamiento, estudios factoriales.

\section{RESUMO}

O processo de integração de três mulheres argentinas na carreira científica é descrito a partir do momento em que eles obtiveram um doutorado em psicologia enquanto estavam trabalhando em um laboratório estrangeiro durante a década de 1960. A trajetória de Hermelinda Fogliatto, Rosalía Paiva e Ana María Insúa é retratada revelando sua chegada da educação ao campo da psicologia. Enquanto não havia tradição de promover o doutorado em psicologia na Argentina, eles conseguiram nesse objetivo sob a direção de Horacio Rimoldi no Laboratório Psicométrico da Universidade de Loyola (Chicago), que as encorajou em suas carreiras. Eles realizaram pesquisas sobre o processo de pensamento para resolução de problemas, com o modelo fatorial, consideradas como referência internacional. Trabalharam 
para organizações apoiadas pelas agências de Saúde e Educação dos Estados Unidos. Duas delas retornaram à Argentina onde trabalharam para o Consejo Nacional de Investigaciones Científicas y Técnicas. Discute-se o papel desta instituição na promoção da pesquisa psicológica bem como o lugar da pesquisa no treinamento e perfil dos psicólogos.

Palavras-chave: História da psicologia, mulheres, carreira científica, processo de pensamento, estudo fatorial.

\begin{abstract}
The integration of three Argentine women in the scientific career is described from the time they obtained a PhD in Psychology while working at a foreign laboratory in the 60's. The auspices under which they were integrated in the scientific career and the public policies which supported them are analyzed to understand how they took advantage from the opportunities they had to produce and manage science. The paths of Hermelinda Fogliatto, Rosalía Paiva and Ana María Insúa are portrayed revealing their arrival from education to the field of psychology. While there was no tradition of promoting PHDs on psychology in Argentina, they achieved this goal at the Psychometric Laboratory of Loyola University (Chicago) under the direction of Horacio Rimoldi, who encouraged their careers. Applying factorial models, they conducted research on the thinking process for problem solving, which was considered work of international reference. These women worked for organizations supported by Health and Education agencies of the United States. Two of them returned to Argentina where they worked for the National Council of Scientific and Technical Research. The role of this institution for the promotion of psychology research is discussed, as well as the place research had in psychologists training and profiles.
\end{abstract}

Keywords: History of psychology, women, scientific career, thinking process, factorial studies.

En el presente trabajo destacaremos la figura de tres mujeres argentinas que se desempeñaron como científicas en psicología: Hermelinda Fogliatto (1925-2003), Rosalía Paiva (1932-1986) y Ana María Insúa (1931-). Durante la década de 1960, cuando se estaban iniciando las carreras de psicología en el país, ellas fueron algunas de las primeras en obtener un doctorado en Psicología, convirtiéndose en investigadoras. Las tres desarrollaron sus estudios doctorales en el Laboratorio de Psicometría de la Universidad de Loyola (Chicago, Illinois, Estados Unidos) bajo la dirección de Horacio Rimoldi, conduciendo pesquisas de referencia internacional sobre procesos de pensamiento para la resolución de problemas. Los peculiares casos de H. Fogliatto, R. Paiva y A. M. Insúa contribuyen a visibilizar algunas características del sistema científico argentino y condiciones para la integración de mujeres a la carrera científica.

En la historiografía internacional posterior a la década de 1980, se ha cuestionado el olvido de las mujeres en la historia de la psicología de países centrales (Furumoto \& Scarborough, 1986; Giménez, 2007; Radtke, Hunter, \& Stam, 2000; Winkler, 2004) y periféricos (Ostrovsky, 2008; Jacó-Vilela, Degani-Carneiro, \& Novaes Messias, 
2012; Winkler, 2015). Buscando reposicionarlas como sujetos de la historia, estos estudios han procurado subsanar la falta de crédito otorgado a ellas en las historias tradicionales.

En esa dirección, con sensibilidad hacia las problemáticas de género, se han reconstruido historias de mujeres eminentes que, entre las primeras generaciones de psicólogos, han tenido que desafiar estereotipos sociales, muchas veces científicamente legitimados (Ostrovsky, 2014), y enfrentar obstáculos mayores para trabajar como científicas (Milar, 2000). Partiendo de la premisa de que la ciencia es una actividad social y que su organización puede ser analizada desde el punto de vista de la división del trabajo, se ha señalado que en Estados Unidos inicialmente la carrera científica ha estado predominantemente asociada a figuras masculinas. Desempeñándose en esferas separadas, la vida pública correspondía a los hombres, mientras que a las mujeres les correspondía la vida privada, cuyas tareas hogareñas de maternidad y crianza eran difícilmente negociables (Furumoto, 1992).

Desde este punto de vista, Sprung y Sprung (1996) han descripto cinco modelos de integración de mujeres al trabajo en el sistema científico en Alemania. En primer lugar, aquellas que han sido auspiciadas como pareja de un académico para el cual trabajan. En segundo término, mujeres que se han integrado al trabajo científico legitimadas por una relación de padre-hija con un académico reconocido. Tercero, mujeres que han sido parte del staff de académicos prominentes, y que luego han desarrollado su propia carrera. Cuarto, mujeres que avanzaron independientemente en su vida profesional luego de haber formado parte de un equipo o grupo académico. Finalmente, mujeres luchadoras solitarias, que han encontrado su propio camino en la vida académica. El análisis de Sprung y Sprung (1996) se basó en diversos indicadores de integración a la vida académica, como: participación en congresos, tesis doctorales, membresías en asociaciones científicas, filiación a grupos de trabajo científico. En consonancia con este análisis de auspicios, estudios locales (Ostrovsky, 2011) han tomado en cuenta las publicaciones de mujeres como indicador de su integración a la carrera científica.

Por otra parte, también se ha descripto la manera en que ciertos movimientos sociales y políticas públicas en torno al bienestar y la educación del niño han brindado oportunidades para el acceso de mujeres a puestos de trabajo en instituciones de salud o educación, donde han podido desarrollarse como investigadoras, e incluso han alcanzado relevancia social en organizaciones científicas por su liderazgo en programas de investigación y en publicaciones (French, 1988).

En efecto, desde la década de 1950 en Argentina también se ha asistido a un progresivo acceso de mujeres a la educación superior y 
feminización de algunas profesiones asociadas a las tareas de cuidar, educar, curar, administrar en las que se proyectan funciones socialmente entendidas como maternas (Falcone, 2015). De hecho, se ha descripto cómo una porción importante de las pioneras argentinas en psicología, ingresaron a la profesión desde la carrera de medicina o de maestra (Winkler, 2007), y se ha señalado que la matrícula de las carreras de psicología ha sido predominantemente femenina (Alonso, 1999).

La historiografía argentina ha rescatado figuras de psicólogas que rompieron con los estereotipos de la época para acceder a la formación científica y a las oportunidades para producir y gestionar ciencia, y alcanzar visibilidad social (Carli, 2016). Se han retratado perfiles de pioneras de la primera mitad del siglo XX (ej. Ostrovsky, 2008; Rodríguez Sturla, 2001a, 2001b), se ha trazado un panorama general de mujeres psicólogas y psicoanalistas entre la década de 1940 y 1970 (Winkler \& Reyes Espejo, 2014), y se han destacado algunas mujeres que contribuyeron a la organización de las carreras de psicología (Ostrovsky \& Moya, 2013). Pero muy pocos trabajos analizaron aportes de mujeres a la investigación en el período inicial de la profesionalización de la psicología (Ferrero, lemme Khoury, Todisco, \& Scherman, 2016; Piñeda \& Scherman, 2016a), o se centraron en el proceso de integración de mujeres a la carrera científica en ese período (Piñeda, n.d.; Piñeda \& Scherman, 2016b).

En el presente trabajo, procuramos subsanar la omisión de $\mathrm{H}$. Fogliatto, R. Paiva y A. M. Insúa de la historiografía local tradicional y nos proponemos realizar una presentación de sus figuras rescatando algunos de sus aportes a la investigación en psicología.

Siguiendo las fases de la investigación histórica descriptas por Klappenbach (2014), explicitamos las herramientas metodológicas en que nos basamos. En primer lugar, acotamos nuestro objeto a la descripción de la integración de estas tres mujeres a la carrera científica en el período inicial de la profesionalización de la psicología argentina a partir de la realización de sus estudios doctorales en un laboratorio extranjero. Problematizamos cuáles fueron los auspicios personales y los apoyos en políticas públicas que les otorgaron oportunidades para producir y gestionar ciencia. Desde una historia local de perspectiva policéntrica (Brock, 2014) valoramos las interrelaciones entre los centros académicos de diferentes países en cuyas comunidades ellas se integraron. Para ello se construyó un estudio de diseño ex post facto retrospectivo de tipo historiográfico (Montero \& León, 2005) en el que se reunió un corpus documental y testimonial sobre datos familiares y académicos de $\mathrm{H}$. Fogliatto, R. Paiva y A. M. Insúa. Estos datos fueron analizados según indicadores de integración a la carrera científica y de auspicios construidos a partir de los aportes de French (1988), Furumoto (1992), Ostrovsky (2011) y Sprung y Sprung (1996). En ese sentido, consideramos: 
situación familiar, edad de inicio de las tres mujeres en los estudios de grado, de posgrado y de empleo científico, titulación alcanzada, cargo, filiación institucional, grupo de trabajo, redes académicas, financiamiento $y$, por último, visibilidad académica lograda en las publicaciones.

Para el relevamiento documental, se ingresó al repositorio digital de textos completos de tesis y disertaciones de la Universidad de Loyola almacenadas electrónicamente como Thesis and Dissertations at Loyola eCommons registradas por año, autor, área temática, entre otros factores. Las tesis constaban de una breve referencia biográfica de sus autores. Se buscaron todas las tesis en las que $\mathrm{H}$. Rimoldi fue mencionado (por ejemplo, en la carátula, agradecimientos, autoridades firmantes, referencias, etc.), recuperando un total de 42 tesis que cumplían esta condición. Estas fueron descargadas, listadas, analizadas y clasificadas. Se seleccionaron para el análisis las 35 tesis que constatamos que fueron dirigidas por $\mathrm{H}$. Rimoldi. Estas fueron publicadas entre 1959 y 1970 . Registramos que 11 de ellas eran de autoría femenina: 6 de nacionalidad estadounidense, 3 argentinas, 1 portoriqueña y 1 india. En tanto los autores masculinos eran: 22 estadounidenses, 1 italiano, 1 canadiense. Las 3 tesis de autoras argentinas correspondieron a H. Fogliatto, R. Paiva y A. M. Insúa. Estas fueron analizadas en profundidad.

Para el análisis de su integración a la carrera científica, recurrimos a los pocos registros escritos públicos sobre la biografía y trayectoria de estas tres mujeres que se conservan, y cuando ello resultó insuficiente, procuramos testimonios de informantes clave. Principalmente consultamos a tres investigadores que, por más de veinte años, fueron colaboradores de $\mathrm{H}$. Rimoldi en el equipo de trabajo que él organizó en el Centro Interdisciplinario de Investigaciones en Psicología Matemática y Experimental (CIIPME): los doctores Eduardo Moreno, Carla Sacchi y Cristina Richaud. Se relevaron fuentes que mencionan a $\mathrm{H}$. Fogliatto, R. Paiva y $\mathrm{A}$. M. Insúa (Altamirano, Clark, \& Stabile, 2012; Ale, 2017; Piñeda, 2012; Piñeda \& Scherman, 2016a), entre las que se destaca el relato autobiográfico de H. Rimoldi (1995), y buscamos obituarios de ellas (Pérez \& Passera, 2004; Seglares Claretianos, 2005). Por otra parte, rastreamos y construimos un listado de las publicaciones de estas tres mujeres en revistas científicas y libros desde los años en que realizaron sus estudios doctorales hasta la fecha de deceso o jubilación. Mediante tales publicaciones accedimos a información sobre filiaciones institucionales, cargos y financiamientos. Las coautorías permitieron indagar datos sobre sus colaboradores, grupos de trabajo y redes académicas.

Ponderamos los doctorados realizados por $\mathrm{H}$. Fogliatto, R. Paiva y A. M. Insúa en el contexto localy la elección que ellas realizaron del Laboratorio de Psicometría de la Universidad de Loyola como ámbito 
de trabajo. Para ello, se revisaron fuentes secundarias sobre el papel de los laboratorios de psicología en Argentina en el contexto sudamericano, así como fuentes primarias y secundarias sobre académicos argentinos que realizaron su doctorado en Psicología antes y después de la creación de las carreras de Psicología en Argentina. Tuvimos en cuenta la cantidad de hombres y de mujeres doctorados hasta 1970, los laboratorios en los que estos trabajaron, sus tradiciones académicas y líneas de investigación, las instituciones oficiales promotoras y/o financiadoras de las mismas.

Los resultados son presentados en dos partes. Primero, se contextualiza la psicología científica en Argentina a mediados del siglo $X X$. Se ofrece un panorama del papel de los laboratorios de psicología argentinos en contexto sudamericano durante la primera mitad del siglo XX, las comunidades que se constituyeron en torno a estos y sus tradiciones académicas. Se presentan hombres y mujeres argentinos doctorados en psicología antes y después de la creación de las carreras de psicología. Se destacan los laboratorios en que trabajaron, financiamientos y redes académicas. En ese sentido, se profundiza en la trayectoria de $\mathrm{H}$. Rimoldi que contribuye a comprender la integración de H. Fogliatto, R. Paiva y A. M. Insúa a la carrera científica, así como sus aportes a la investigación. En el segundo apartado, se describe la trayectoria de estas tres mujeres. Sus orígenes y aspectos familiares, edad y modo de acceso a la psicología, apoyos para el ingreso a la carrera científica, instituciones que las financiaron, línea de investigación a la que contribuyeron mediante su tesis y publicaciones, e incidencia de estas. Se concluye comparando el modo en que H. Fogliatto, R. Paiva y A. M. Insúa accedieron desde el campo educativo a la psicología integrándose a la carrera científica. Finalmente, se discute el rol del Consejo Nacional de Investigaciones Científicas y Técnicas (CONICET) en las políticas públicas de promoción de la investigación en psicología, el lugar de la misma en las carreras universitarias y su incidencia en el perfil de psicólogo.

\section{La psicología científica en Argentina a mediados del siglo XX}

Para dimensionar la opción de una mujer, en Argentina durante la década de 1960, para acceder a la carrera científica mediante un doctorado en psicología en un laboratorio extranjero como ámbito de trabajo, procuramos describir algunos elementos de ese contexto.

En diversas culturas académicas, el laboratorio de psicología ha sido considerado un símbolo de desarrollo científico. Al iniciar el siglo XX en Argentina, como en otros países sudamericanos, se asistía a la creación de laboratorios de psicología experimental que han marcado un hito de institucionalización de la disciplina. Estos han representado 
el progreso al abrigo de Estados modernos que, bajo los ideales del positivismo, propendían a la organización científica de las instituciones en temas sociales como educación, salud y justicia. Muchas veces, los laboratorios fueron organizados con fines didácticos, asociados a cátedras universitarias 0 en escuelas normales. Sin embargo, paulatinamente éstos se convirtieron en organismos productores de ciencia básica y aplicada y contribuyeron a la constitución de comunidades científicas. En un principio, fueron montados con aparatos importados de Francia, Bélgica o Alemania, y posteriormente fueron incorporando otro tipo de instrumentos de medición basados en procedimientos estadísticos, dando cuenta de modelos ingleses y estadounidenses (Centofanti, 1982; Dias Cirino, Lopez Miranda, \& De Souza Júnior, 2012; Ferrari, 2013; Klappenbach, 2006; López Miranda, Rota Júnior, Baker, \& Dias Cirino, 2016; Miguez, Betancourt Mainhard, \& Villarroel, 2010; Prado da Fonseca, Rocha Silva da Rosa, \& Arruda Leal Ferreira, 2016; Salas Contreras, 2012).

Las principales tradiciones científicas sucesivamente sedimentadas en la psicología argentina de la primera mitad del siglo XX, pueden verse representadas en doctorados registrados en el período, a su vez ligados a grupos de trabajo y redes académicas frecuentemente organizados en torno a laboratorios. Antes de la organización de las carreras de Psicología en el país, argentinos que alcanzaron ese nivel de estudios en la disciplina lo hicieron en el exterior.

El primer doctorado en Psicología habría sido Leonardo Castellani (1899-1981), quien obtuvo su título en 1934 en La Sorbonne, auspiciado por la Compañía de Jesús, comunidad de la que formaba parte en esa época (Piñeda, 2005). Fue dirigido por George Dumas (1866-1946). El maestro francés -que había visitado la Facultad de Filosofía y Letras de la UBA en tres oportunidades (1908, 1918, 1920) (Falcone, 2012), era Jefe del Laboratorio de Psicología Clínica de Enfermedades Mentales de la Facultad de Medicina de París en el Hospital Sainte-Anne. La tesis de Castellanide notaba influencias frecuentes en la psicología argentina de la época: comportamentalismo (Dagfal, 2002; Klappenbach, 2006) y psicología experimental franceses (Piñeda, 2014), y la tradición experimental de la Escuela de Lovaina (Piñeda, 2007).

En 1949, Horacio Rimoldi (1913-2006) sería el segundo en obtener su título doctoral en Psicología. Él había estudiado Medicina en la Universidad de Buenos Aires (UBA), siendo discípulo y colaborador de Bernardo Houssay (1887-1971). El Premio Nobel en Fisiología de 1947 y primer presidente de CONICET (1958-1971), reiteradas veces materializó el apoyo a su carrera, impulsándolo para viajes al exterior y retornos al país. En su paso por Inglaterra entre 1939 y 1941, Rimoldi se inició en Oxford en los métodos factoriales con el discípulo de Charles Spearman (1863-1945), William Stephenson (1902-1989), 
reuniendo el material de la tesis doctoral sobre ritmo y fatiga que defendería en Buenos Aires en 1945.

En un período en el que en Argentina se registraba creciente interés hacia las prácticas psicotécnicas orientadas a la educación y al trabajo y comenzaban a circular textos de autores europeos y estadounidenses sobre estos temas (Flores-Mendoza, Ardila, Gallegos, Sampaio Braga, Miranda Carvalhais, \& Andrade, 2016; Klappenbach, 2001), Rimoldi permaneció en el país dirigiendo el Laboratorio de Psicología Experimental de la Universidad Nacional de Cuyo (1941-1946), donde junto a su discípula Nuria Cortada (19212013) llegó a realizar la primera estandarización extranjera de matrices progresivas de Raven. A continuación, Cortada se dirigió a Estados Unidos, donde obtuvo en 1948 su Master of Arts en Psicología Clínica por la Universidad del Estado de Ohio. Así, se convirtió en la primera psicóloga de profesión en Argentina, y colaboró en la organización del primer plan de estudios de dicha carrera en la UBA (Cortada, 2006).

En 1946, Rimoldi también viajó y se incorporó al Laboratorio de Psicometría de la Universidad de Chicago, colaborando con Louis Thurstone (1887-1955) y profundizando sus conocimientos en análisis factorial. Durante 1947 trabajó en Harvard sobre "Personal Tempo", vinculándose con figuras de renombre mundial como Gordon Allport (1897-1967), Edwin Boring (1886-1968), y con el francés Paul Fraisse (1911-1996) que estudiaba sobre ritmo (tema relacionado a tempo). En 1948 regresó a Chicago y desarrolló su tesis para obtener el PhD en Psicología al año siguiente. Su "Estudio de algunos factores relacionados a la inteligencia" utilizó buena parte del material obtenido en Mendoza. En esa etapa inició amistad con el conocido factorialista Raymond Cattell (1905-1998) de la Universidad de Illinois. Continuando con sus investigaciones sobre el proceso de resolución de problemas, Rimoldi desarrolló una técnica para el estudio de los procesos cognitivos y tácticas empleadas durante ese proceso, que primeramente ejemplificó en el Test de Habilidades Diagnósticas, pero que luego tuvo aplicaciones en diversos ámbitos. En 1955 Rimoldi se estableció en la Universidad de Loyola donde fue designado Profesor Distinguido y organizó el Laboratorio de Psicometría. Allí llegó a dirigir numerosas tesis de doctorado entre las que se encuentran las de las tres mujeres que describiremos. En esos años además viajó a diferentes países como Profesor Invitado, pasando por Colombia y Uruguay, donde también fundó un laboratorio en la Universidad de La República. Desde 1957 Rimoldi realizó algunas estancias como profesor invitado en la carrera de Psicología de la Universidad Nacional de Córdoba (UNC), y asesoró investigaciones en psicoacústica del Centro de Investigaciones Acústicas y Luminotécnicas $(\mathrm{Cl} A \mathrm{~L})$ de esa universidad. Pero fue recién en 1970 cuando, promovido por Houssay, regresó a Buenos Aires. La 
misión encomendada fue la organización del Centro Interdisciplinario de Investigaciones en Psicología Matemática y Experimental (CIIPME) de doble dependencia: Facultad de Medicina de la UBA-CONICET (Rimoldi, 1995).

En 1954, Miguelina Guirao fue la tercera académica en obtener su doctorado, en Psicología Experimental, en la Universidad Católica del Sagrado Corazón (Milán). Realizó estudios sobre procesos cognitivos en el Laboratorio de Psicología bajo la dirección de Agostino Gemelliligado a la tradición experimental lovainense- cuyos trabajos sobre percepción, lenguaje, personalidad, psicología evolutiva, social e industrial, eran muy populares en Argentina. Luego de un fugaz paso como docente de psicología experimental por la flamante carrera de psicología de Rosario, Guirao obtuvo una beca posdoctoral de CONICET entre 1962 y 1963 para estudiar psicoacústica en el Laboratorio de Psicofísica de Harvard con S. S. Stevens (1906-1973). Este destino le fue recomendado por B. Houssay. Al regresar al país, CONICET la incorporó como Investigadora para organizar el Laboratorio de Investigaciones Sensoriales (LIS) en la Facultad de Medicina de la UBA, y también mantuvo vínculos con el $\mathrm{ClAL}$ y el CIIPME (Piñeda \& Scherman, 2016b).

También en la Universidad Católica del Sagrado Corazón, en 1964 se doctoró Carla Sacchi con su Tesis sobre la aplicación de una técnica de grupo al tratamiento de alcohólicos crónicos (Sacchi, 1963). Ella se había graduado de Profesora de Enseñanza Secundaria Normal y Especial en Pedagogía y Filosofía de la Universidad Nacional de Cuyo (San Luis) en 1960 (Universidad Nacional de Cuyo, n.d.). En Argentina, se integró al sistema científico con Beca de CONICET (1969-1971) y Beca de la Fundación Argentina de Salud Mental (1972-1973) trabajando en la Cátedra de Psicología Médica del Hospital de Clínicas de la UBA. Luego, ingresó a la carrera de Investigador de CONICET en el CIIPME como lugar de trabajo. Se desempeñó como docente de grado y de posgrado en diversas universidades del país. Fue socia fundadora de la Asociación Argentina de Ciencias del Comportamiento (AACC) y miembro de la Asociación Argentina de Estudios e Investigaciones en Psicodiagnóstico (ADEIP).

Tras la creación de las carreras de Psicología en Argentina, entre 1961 y 1963 se graduaron los primeros licenciados de las universidades pioneras. Entre las carreras que en su plan de estudios contaban con el nivel de doctorado, registramos en San Luis esa primera titulación, obtenida en 1965 por Rafael Ramón Rossell, con una tesis sobre la hipnosis (Universidad Nacional de San Luis, n.d.). Le siguió Eva Borkowska de Mikusinski (1921-) que, con beca de CONICET, realizó su trabajo de tesis doctoral en la Universidad de Londres bajo la dirección de Hans Eysenck (1916-1997) entre 1964 y 1965, y la defendió en San Luis en 1969, donde continuó trabajando 
hasta su jubilación como investigadora de CONICET. En Argentina fue introductora de los estudios factoriales de la personalidad de Eysenck, modelo al que integraba los aportes del ya mencionado R. B. Cattell. También realizó estancias posdoctorales donde profundizó aspectos motivacionales del comportamiento en la Universidad de Michigan con Robert Kastenbaum (1933-2013) y en el Laboratorio de Psicología Experimental de la Universidad de René Descartes con Paul Fraisse (Piñeda, n.d.). Otra colega sanluiseña, Claribel Morales de Barbenza (1932-2017), le siguió con similar recorrido. También con beca de CONICET, entre 1966 y 1968, estudió en la Unidad de Audiología del Departamento de Ingeniería Eléctrica, Universidad de Salford (Inglaterra), dirigida por William Tempest. Allí obtuvo su Master of Sciences, y defendió su tesis doctoral en San Luis en 1971. Su trabajo en psicoacústica dialogaba con los aludidos estudios de S. S. Stevens y de M. Guirao (Piñeda \& Scherman, 2016a).

Estos datos empíricos sugieren que, hasta 1960 en Argentina no había tradición de realizar doctorado sen Psicología y que, hacia finales de esa década, las mujeres doctoradas duplicarían a los hombres, insertándose en diversos laboratorios para lograrlo.

Con todo, la tradición más extendida en la psicología argentina desde 1960 ha sido el psicoanálisis, que se expandió en las carreras de psicología (Dagfal, 2018), bajo la incidencia de médicos de la Asociación Psicoanalítica Argentina en los planteles docentes. La difusión del psicoanálisis en este período fue abonada por los cambios económicos y culturales sucedidos tras el derrocamiento del gobierno peronista en 1955. Allí donde la planificación estatal había promovido la psicotecnia y la orientación profesional -con sus prácticas colectivas de administración de tests-, en nombre de la modernización floreció la psicoterapia psicoanalítica al servicio de la individualidad de los sujetos. Se provocó un vaciamiento de los fundamentos científicos de la psicología y de prácticas experimentales conocidas hasta el momento. Dicho lugar fue ocupado por el psicoanálisis, que se convirtió en el esquema referencial de la psicología, promoviendo la formación de psicólogos de perfil clínico para el ejercicio liberal de la profesión. (Klappenbach, 2018). Rubén Ardila (1979) se refirió a este hecho peculiar sosteniendo que dos factores impedían el desarrollo de la psicología experimental en Argentina. El primero, era el avance masivo del psicoanálisis. En opinión de Ardila, esto se debía a que las carreras de psicología estaban dominadas por médicos psicoanalistas que limitaban el entrenamiento de los psicólogos, confundían psiquiatría, psicoanálisis y psicología, y operaban para que los médicos tuvieran supremacía sobre los psicólogos en el ejercicio de la profesión. El segundo factor se refería al contexto de crisis política y la ideologización de las carreras de psicología por la cual se asociaba psicología experimental a "imperialismo" o "positivismo lógico", una práctica "contraria a la liberación nacional" (Ardila, 1979, p. 86). Por 
estas razones, Ardila reconocía muy pocos centros de investigación experimental en el país al finalizar la década de 1970, entre los que se encontraban los de San Luis, LIS y CIIPME, antes referidos.

Asimismo, otro factor se agrega para explicar el escaso desarrollo en el país de las tradiciones experimentales en sus versiones europeas latinas y anglosajonas- 0 norteamericanas. El progresivo desfinanciamiento de las carreras de psicología, más evidente en las de matrícula elevada como las de Buenos Aires, La Plata o Córdoba, donde el presupuesto era prácticamente absorbido en cobertura del plantel docente (Chaparro, 1969), limitando toda inversión en infraestructura requerida para investigación.

En consecuencia, pocos de los académicos doctorados mencionados se integraron al plantel docente de una carrera de psicología. Se ha comprobado que hasta la década de 1980los laboratorios e institutos que absorbieron a psicólogos abocados a estudios experimentales estaban frecuentemente vinculados al campo bio-médico y ajenos a las carreras de psicología (Piñeda, 2012). Desde su creación en 1958, CONICET fue promoviendo con becas e incorporaciones a la carrera de investigador este tipo de perfiles, como lo demuestran los casos de Guirao, Sacchi, Mikusinski, Barbenza y el mismo Rimoldi. En ellos se evidenciaba cercanía a modelos anglosajones.

\section{Tres mujeres: Hermelinda Fogliatto, Rosalía Paiva y Ana María Insúa}

Hermelinda Fogliatto nació en Santiago Temple, un pequeño pueblo a $80 \mathrm{~km}$ de la ciudad de Córdoba el 4 de julio de 1925. Graduada en 1943 en la Escuela de Maestras Alejandro Carbo, enseñó en diferentes escuelas públicas de la provincia hasta 1957 (Fogliatto, 1963).

Ese año, H. Rimoldi estuvo en Argentina. Había interrumpido su estancia en Loyola al ser designado Profesor Titular de Psicología II en la recientemente organizada carrera de dicho campo en la UBA, pero desacuerdos políticos determinaron que renunciara (Rimoldi, 1995). Sin embargo, ese mismo año Rimoldi fue contratado como Profesor Extraordinario por la Facultad de Filosofía y Humanidades de Córdoba que también había organizado su carrera de Psicología. Se lo había convocado para dictar Psicoestadística y asesorar investigaciones. En esa oportunidad, lazos duraderos se fueron entablando para fructificar en diversos proyectos. Entre ellos, Rimoldi se vinculó con el equipo dirigido por el Ingeniero Guillermo Fuchs en el CIAL (Facultad de Arquitectura), que a su vez durante la década de 1960 se relacionó con docentes y egresados de la carrera de Psicología para trabajos interdisciplinarios (Piñeda \& Scherman, 2016a). Fogliatto estableció contacto con Rimoldi y obtuvo la 
admisión en la Escuela de Graduados de la Universidad de Loyola para iniciar allí sus estudios de posgrado en septiembre de 1958. Tras dos años de estudios en el Departamento de Educación, en junio de 1960 obtuvo su Master en Educación. Desde ese momento, se desempeñó como investigadora en el laboratorio de psicometría dirigido por Rimoldi. También bajo su dirección, y con el financiamiento del Programa de Investigación Corporativa de la Oficina de Educación del Departamento de Salud, Educación y Bienestar de Estados Unidos, Fogliatto realizó su tesis de doctorado con el tema: "Diferencias individuales y procesos de pensamiento", que defendió en 1963 (Fogliatto, 1963). Como fruto de ese trabajo, contribuyó al desarrollo de la técnica de evaluación del proceso dinámico de resolución de problemas, mediante la identificación de la secuencia de preguntas (táctica) que el sujeto se formula para resolverlo, analizando su dificultad según las relaciones lógicas y lenguaje de formulación utilizado, así como la incidencia del entrenamiento en el proceso. Su tesis y otras contribuciones vinculadas a ella (ej. Rimoldi, Haley \& Fogliatto, 1962; Rimoldi, Fogliatto, Haley, Reyes, Erdmann \& Zacharia, 1964) robustecieron las hipótesis y la técnica de Rimoldi, y fueron referencia central para otras 11 tesis realizadas en el Laboratorio de Psicometría de Loyola en el período estudiado.

Retornando a Argentina, a partir de 1965, Fogliatto se incorporó como investigadora de CONICET y se unió al plantel docente de la carrera de Psicología de Córdoba. Allí estuvo a cargo de la enseñanza de "Métodos y técnicas de investigación" y "Orientación y selección Profesional", con la colaboración del italiano Franco Murat (Altamirano, Clark \& Stabile, 2012), que había formado parte del equipo docente y del Instituto de Investigaciones Psicopedagógicas en San Luis (Piñeda, n.d.). También junto a F. Murat, H. Fogliatto asesoró numerosas investigaciones, incluidas varias de las que se desarrollaron en el ClAL (Piñeda \& Scherman, 2016b). Desde un modelo actuarial, fue una de las pioneras en la orientación vocacional en Argentina. Cuenta con unas 40 publicaciones editadas por la UNCsobre predicción de rendimiento de estudiantes, con profesiogramas de todas las carreras que se enseñaban en esa universidad. Colaboró con el análisis factorial de diversas pruebas y la construcción de cuestionarios de intereses profesionales, y desarrolló un programa informático de orientación vocacional (Fogliatto, 1999).

En diversas circunstancias, los aportes científicos de Fogliatto entraron en conflicto con la cultura académica dominante en las carreras de psicología del país de sesgo profesionalista, clínico, psicoanalítico, a veces permeable a discursos políticos radicalizados, y resistente a la psicología experimental. Su modelo de orientación vocacional registró tensiones con el modelo clínico psicoanalítico desarrollado por Rodolfo Bohoslavsky, que también había sido 
eclécticamente incorporado en Córdoba (Altamirano, et al., 2012). Bohoslavsky fue sucesor de Nuria Cortada en la Dirección del Servicio de Orientación Vocacional de la UBA que ella había organizado en 1958.

A mediados de 1970, a estas tensiones se sumaron resistencias ideológicas de estudiantes y docentes de la carrera de Psicología de Córdoba que, desde un discurso de izquierda, difundían consideraciones adversas hacia lo que entendían como psicología "pro-yanqui". En general, así eran calificados los enfoques experimentales, cuantitativos, de metodología rigurosamente estadística. Por este motivo, algunos estudiantes protestaron contra Fogliatto (Ale, 2017) Con todo, la docente también logró una buena repercusión entre jóvenes a los que formó como investigadores como: Ana María Rovere, Josefina Passera, Ana María Alderete, María Isabel Oberto, Marta Bruno, Livio T. P. Grasso y Emilia Rojo, los dos últimos más tarde integrados al CIIPME.

La dedicación de Fogliatto a la ciencia se complementaba con su consagración al movimiento de seglares claretianos. Soltera y de buena posición económica (Ale, 2017), en dicho movimiento fundó una guardería para niños de familias carenciadas. Falleció a los 78 años en el año 2003 (Pérez \& Passera, 2004; Seglares Claretianos, 2005).

La técnica de evaluación de procesos de resolución de problemas que Fogliatto contribuyó a desarrollar junto a Rimoldi, fue una pieza clave en el trabajo doctoral elaborado por la cordobesa Rosalía Paiva sobre "Tiempo y proceso de resolución de problemas". La investigación, que se realizó con una muestra de estudiantes universitarios cordobeses, utilizando cuatro problemas verbales y uno gráfico de dificultad variable, demostraba la hipótesis de que el rango de tiempo en el que un sujeto formula preguntas (tácticas de resolución de problemas), es a intervalos constantes. Se administraron seis pruebas sobre tiempo de reacción en movimiento muscular, percepción, lectura y dibujo, para analizar si existen relaciones entre estos factores y el tiempo de resolución de los problemas, y cómo se relacionan. Se buscaron relaciones entre táctica ideal, buena y mala, grupo más rápido; tiempo de comprensión del problema (primera pregunta), y consistencia interna en cada sujeto en relación a la resolución de los problemas (Paiva, 1968).

Nacida en la capital cordobesa el 17 de junio de 1932, con 29 años fue una de las primeras graduadas del Profesorado en Psicología y Pedagogía en 1961. El mismo se había organizado en 1956, dos años antes de que el plan de estudios de la licenciatura fuera oficializado, y en ese contexto en 1957 Paiva fue alumna de Rimoldi (1995). El pasaje de Paiva desde las aulas de Psicología a los laboratorios de esta disciplina se inició con sus estudios de posgrado en Francia entre 1963 y 1964 donde realizó una estancia en la Universidad de Paris y 
en la Escuela Práctica de Altos Estudios. Al año siguiente, fue admitida en Loyola donde se desempeñó como investigadora asistente en el Laboratorio de Psicometría dirigido por Rimoldi desde febrero de 1965 hasta junio de 1966. A partir de ese momento, fue investigadora asociada en el Centro de Investigación y Entrenamiento sobre Enfermedades Congénitas del Corazón del Instituto Hektoenpara la Investigación Médica (Paiva, 1968) dirigido por Dr. Maurice Lev, MD (Lev, Rimoldi, Paiva \& Arcilla, 1969). Trabajó como investigadora para el Departamento de Patología de la Escuela de Medicina de la Universidad del Noroeste; el Departamento de Pediatría de la Escuela de Medicina de la Universidad de Chicago, y para el Colegio de Medicina de la Universidad de Illinois. Permaneció en Illinois hasta su muerte en 1986 (Dawson-Saunders \& Paiva, 1986). En su tesis la autora dejó testimonio del apoyo recibido por sus padres e hijo para concretar su proyecto de vida científica.

El trabajo doctoral de Ana María Insúa, presentado en Loyola en enero de 1970, se complementó con las de las dos antecesoras cordobesas. También dirigida por Rimoldi, abordó “Algunas variables de personalidad en relación al proceso de resolución de problemas de estudiantes secundarios". Las variables de personalidad fueron exploradas en 152 sujetos mediante el test de manchas de Holtzman, en relación a problemas de movimiento, color, forma y ubicación en base al método de Rimoldi (Insúa, 1970).

A.M. Insúa nació el 28 de octubre de 1931 en la santafesina ciudad de Casilda, a $71 \mathrm{~km}$ de Rosario. Era nieta de inmigrantes españoles de parte de su padre, e italianos de parte de su madre. Su padre murió muy joven. Su madre realizó grandes esfuerzos para dar estudios superiores a cuatro hijos: tres varones (dos ingenieros, un médico) y una mujer (Insúa, 2013). En sintonía con varios de su generación, que trabajaron como primeros docentes de las carreras de psicología argentinas, A. M. Insúa se graduó como Profesora de Enseñanza Secundaria Normal y Especial en Filosofía en la Universidad de Buenos Aires (UBA) (Insúa, 1970).

A los fines de este relato, es importante destacar que el hermano médico era el Dr. Jorge A. Insúa (Dr. Eduardo Moreno, comunicación personal 17/12/18), desde 1961 a cargo de la Cátedra de Psicología Médica de la Facultad de Medicina de la UBA con sede en el Hospital de Clínicas. Ese grupo fue impulsor de la Fundación Argentina para la Salud Mental (FASAM) que a su vez editaba la revista Psicología Médica. Con beca del Consejo Británico (1952-1954), J. Insúa había realizado estudios sobre Psiquiatría Clínica, Psicología Médica y Neuropatología en el Hospital Maudsley (Londres). En 1990, llegó a ser miembro de la Academia de Medicina, donde presidió el Consejo de Certificación de Profesionales Médicos (Insúa, 2013).

Antes de partir a Loyola, A. M. Insúa había realizado sus primeras labores profesionales como psicóloga clínica en el Instituto John H. 
Jackson en Buenos Aires. También se dedicó a la docencia universitaria, enseñando el test de Rorschach en el Instituto Superior de Educación Religiosa y en la Universidad Católica Argentina (Insúa, 1970). Esta universidad inició su carrera de Psicología en 1961 (Piñeda, 2007). En 1966, ella tuvo la oportunidad de comenzar sus estudios doctorales en Loyola habiendo obtenido financiamiento de CONICET que la becó durante un año para estudiar metodología de la investigación. Por otra parte, del Estado de Illinois también obtuvo financiamiento del Área de Entrenamiento e Investigación en Psiquiatría del Departamento de Salud Mental. Sus estudios doctorales la llevaron a especializarse en la evaluación de la personalidad con estudios factoriales y correlaciones de resultados entre los tests de Rorschach y Holtzman (ej. Insúa, 1981; Insúa \& Loza, 1986, 1988) que, de alguna manera reorientaban temas clínicos populares en Argentina por esa época.

De regreso a su país en la misma época que Rimoldi, A. M. Insúa se incorporó como investigadora de CONICET en el plantel del CIIPME (Dra. Carla Sacchi, comunicación personal 18/12/18), publicando en su Boletín (Insúa, Rimoldi \& Erdman, 1972) y otros medios (Insúa, 1981; Insúa \& Loza, 1986, 1988; Rimoldi \& Insúa, 1976; Rimoldi, Insúa \& Erdman, 1975). Sus aportes fueron retomados por Sacchi (1977).

Durante las décadas de 1970 y 1980, Insúa también integraba el grupo de Psicología Médica de la UBA y FASAM, dictando cursos de Estadística y Diseño de Experimentos y publicando en la revista (Insúa, 1975). Más tarde, condujo proyectos en el Centro de Investigaciones en Antropología Filosófica y Cultural (CIAFIC), donde trabajó sobre la concordancia en el diagnóstico de depresiones entre el DSM-IV y el SCID-CV y sobre la adaptación y estandarización del Wechsler Adult Intelligence Scale (WAIS-R) a la Argentina.

\section{Conclusiones}

La carrera de Psicología en Argentina ha presentado una matrícula predominantemente femenina (Alonso, 1999) reflejando una tendencia cultural a asociar el rol de psicólogo con la función atribuida a las mujeres de cuidar, curar o educar (Falcone, 2015). Quizás esta haya sido una razón por la que el acceso de mujeres a la psicología muchas veces se haya abierto a partir del campo educativo (Winkler, 2007). Otra de las razones, a mediados de la década de 1950, fue el auge de la psicotecnia en el campo educacional que abrió el camino a los primeros estudios sobre la inteligencia realizados en el país, como los de H. Rimoldi y N. Cortada. Justamente, en ese conjunto de intereses se produjo el acercamiento de H. Fogliatto, R. Paiva y A. M. Insúa a la psicología. 
En efecto, por su fecha de nacimiento entre 1925 y 1931, las tres participaron de la misma generación de mujeres que encontraba frecuentes oportunidades de trabajo como educadoras, razón por la que entre mediados de 1940 y fines de 1950 tuvieron una formación inicial como Maestra o Profesora (Psicología y Pedagogía / Filosofía), en dos de los casos ejerciendo la profesión docente. Oriundas de localidades provincianas, dejaron las aulas para ingresar al laboratorio extranjero y emprender estudios psicométricos de los procesos cognitivos involucrados en la resolución de problemas.

La integración de las tres a la carrera científica fue promovida por $\mathrm{H}$. Rimoldi, quien las dirigió durante el doctorado en el Laboratorio de Psicometría de la Universidad de Loyola (universidad jesuita que no les resultaría indiferente al menos a dos de las mujeres vinculadas a organizaciones católicas). En términos de lo que Sprung y Sprung (1997) definen como modelo de integración en el plantel de un académico reconocido, las tres psicólogas fueron auspiciadas por el jefe de un equipo de trabajo que gozaba de prestigio internacional. Su trayectoria vinculaba las tradiciones factoriales de Inglaterra (C. Spearman y W. Stephenson) y de Estados Unidos (L. Thurstone y R. Cattell) a las que aportaba una línea de investigación original, fructífera y generosa en la formación de investigadores de diversas latitudes. Mediante esta línea de investigación, en tres momentos de su carrera, Rimoldi también conectó varias instituciones argentinas con la psicología producida en aquellos centros extranjeros. Primero en Cuyo, donde se formó inicialmente N. Cortada (2006) y le siguieron C. Sacchi, E. Mikusinski, y C. Barbenza, entre otros (Piñeda, 2010). Luego en Córdoba, de donde surgieron H. Fogliatto, R. Paiva, y tantos otros en la carrera de Psicología y el CIAL (Ferrero et al., 2016). Finalmente, en Buenos Aires, donde ingresó a CONICET, creó el CIIPME, se relacionó con el LIS y en general con la ciencia psicológica que se fue desarrollando en décadas posteriores.

En épocas en que la psicología iniciaba su proceso de profesionalización en Argentina y no existía tradición de cursar doctorados en esa disciplina, H. Fogliatto, R. Paiva y A. M. Insúa se ubicaron entre las primeras mujeres del país en lograrlo, contribuyendo a duplicar en número a los hombres que habían obtenido tal titulación. En Loyola se contaron entre las 11 mujeres que, entre 1959 y 1970, representaron la tercera parte de los doctorados dirigidos por $\mathrm{H}$. Rimoldi.

Accedieron a su primer trabajo como investigadoras entre los 33 y los 35 años. Hasta donde sabemos, una de ellas siendo soltera y de buena posición económica, la segunda a cargo de un hijo y con el apoyo de sus padres, y la tercera con apoyo familiar, especialmente de un hermano médico, afín a la psicología y muy bien posicionado en el sistema científico. 
Conforme a los criterios que hemos construido (French, 1988; Furumoto, 1992; Ostrovsky, 2011; Sprung \& Sprung, 1997), confirmamos que las tres mujeres se integraron a la carrera científica de forma exitosa. Constatamos que, a partir de su experiencia en la Universidad de Loyola, accedieron a cargos como investigadoras. $\mathrm{H}$. Fogliatto y R. Paiva obtuvieron su primer puesto en el Laboratorio de Psicometría dirigido por $\mathrm{H}$. Rimoldi. A continuación, R. Paiva se insertó en programas del campo de la Medicina de un instituto y tres universidades de Estados Unidos, no regresando a la Argentina. Por su parte, H. Fogliattoy A. M. Insúa se integraron a la carrera de investigador de CONICET. La primera también ejerció una intensa labor de investigación desde la Escuela de Psicología de la Universidad Nacional de Córdoba, colaborando con la formación de recursos humanos del CIAL y del CIIPME. A. M. Insúa se integró al equipo del CIIPME bajo la dirección de Rimoldi, y también al de FASAM junto a su hermano J. Insúa. En esos ámbitos continuó combinando sus intereses clínicos con el estudio científico de la personalidad. Si bien las tres investigadoras produjeron importantes publicaciones, H. Fogliatto parece haber sido la más prolífera y de mayor incidencia nacional e internacional.

Aportamos evidencia empírica local a la hipótesis de French (1988) de que en Estados Unidos numerosas mujeres obtuvieron el apoyo de políticas públicas de salud y educación para desarrollar sus carreras como investigadoras. En ese sentido, mientras Fogliatto, Paiva e Insúa permanecieron en ese país, consiguieron financiamiento de agencias oficiales de salud y educación para conducir sus pesquisas. Sus trabajos contribuyeron a fortalecer el de Rimoldi y, en definitiva, un programa en ciencias cognitivas con estudios factoriales, mainstream en el país del Norte y poco popular en Argentina.

En efecto, el laboratorio como figura de prestigio científico asociado a las ciencias naturales, perdía simpatía en las carreras de Psicología locales donde la psicología experimental era quitada de las currículas por el avance del psicoanálisis, ideologías de resistencia a la cultura estadounidense y el desfinanciamiento que limitaba la infraestructura. Sin embargo, dichas investigaciones ganaban terreno en dependencias de CONICET que, desde su creación, había apoyado algunas trayectorias con becas a Inglaterra y a Estados Unidos, así como posteriores ingresos a la carrera científica, subsidios a proyectos y creación de institutos como el LIS y el CIIPME. En esta política se evidenciaba la promoción de una psicología orientada al estudio del comportamiento desde enfoques matemáticos y biológicos (Piñeda, n.d.; Piñeda \& Scherman, 2016a, 2016b). Cabe preguntarse por el arraigo que esas tradiciones tenían en el Presidente de CONICET, B. Houssay, que había estimulado a Rimoldi y a Guirao a institucionalizar estas investigaciones. 
En dicho contexto, tanto Fogliatto como Insúa encontraron alternativas de desarrollo de sus carreras científicas.

No obstante, solo Fogliatto se insertó en una carrera de Psicología de una universidad nacional. Allí debió abrirse camino, pese a las resistencias ideológicas, en un terreno en el que psiquiatría, psicoanálisis y psicología trazaban límites difusos. Esta última se había vuelto una profesión liberal: fundamentada casi exclusivamente desde el psicoanálisis, enfocada hacia la clínica y supeditada a la psiquiatría (Dagfal, 2018; Klappenbach, 2018). Por lo que no había demasiado lugar para estudios factoriales que evocaban anteriores tradiciones que se imponía superar, una cultura foránea que se buscaba combatir y una práctica académica ajena a la profesión que enarbolaba su compromiso con la subjetividad.

La historia de vida y el estudio de la obra de estas tres mujeres que aquí procuramos presentar, sin dudas ameritan nuevos trabajos que profundicen en cada una de ellas. Quedan también interrogantes sobre sus compañeras en el Laboratorio de Loyola y el conjunto total de tesis que allí dirigió $\mathrm{H}$. Rimoldi. Su análisis pormenorizado permitiría ahondar en estudios comparados sobre las líneas de investigación abordadas en los inicios del ClIPME; en otros centros argentinos en los que circuló y se asimiló el modelo factorial, y en universidades de Colombia y de Uruguay donde también actuó Rimoldi (1996) dejando prestigiosas discípulas.

\section{Referências}

Ale, Y. (2017). Injerencias del contexto político en la escuela de psicología de la UNC (1973-76) (Trabajo Integrador Final). Facultad de Psicología de la Universidad Nacional de Córdoba, Córdoba. Recuperado de http: //historiapsicounc. wixsite.com/home

Alonso, M. (1999). Psicología en Argentina. In M. Alonso \& E. Eagly (Eds.), Psicología en las Américas. Caracas: Sociedad Interamericana de Psicología.

Altamirano, P., Clark, C. G., \& Stabile, C. A. (2012). Antecedentes históricos en la Universidad Nacional de Córdoba sobre la orientación vocacional. Década 60/70. IV Congreso Internacional de Investigación y Práctica Profesional en Psicología XIX Jornadas de Investigación VIII Encuentro de Investigadores en Psicología del MERCOSUR. Facultad de Psicología - Universidad de Buenos Aires, Buenos Aires. Recuperado de https://www. aacademica.org/000-072/127.pdf

Ardila, R. (1979). La Psicología en Argentina. Pasado, Presente, Futuro. Revista Latinoamericana de Psicología, 11(1), 71-91. 
Brock, A. (2014). What is a polycentric History of Psychology? Estudos e Pesquisas em Psicologia, 14(2), 646-659. Recuperado de

http://www.epublicacoes.uerj.br/index.php/revispsi/article/view/12565/9749

Chaparro, F. J. (1969). La carrera de psicología en las universidades nacionales. Revista Argentina de Psicología, 2, 147-154.

Carli, S. (2016). Deconstruir la profesión académica: tendencias globales y figuras históricas. Una exploración de las biografías académicas de profesoras universitarias. Propuesta Educativa, 25(45), 81-90. Recuperado de http: //www.scielo.org.ar/pdf/pe/n45/n45a09.pdf

Centofanti, R. (1982). Radecki e a psicología no Brasil. Psicologia: Ciência e Profissão, 3(1), 2-50.

Cortada, N. (2006). Autobiografía. Revista de Historia de la Psicología, 27(4), 49-58.

Dagfal, A. (2002). La naissance d'une 'conduite à la française': de Ribot à Janet. L'Évolution psychiatrique, 67(3), 591-600.

Dagfal, A. (2018). Psychology and Psychoanalysis in Argentina. Politics, French thought and the university connection, 19551976. History of Psychology, 21(3), 254-272. doi: 10.1037/hop0000071

Dawson-Saunders, B., \& Paiva, R. E. (1986). The validity of clerkship performance evaluations. Medical Education, 20(3), 240-245. doi: 10.1111/j.1365-2923.1986.tb01175.x

Dias Cirino, S., Lopez Miranda, R., \& De Souza Júnior, E. J. (2012). The laboratory of experimental psychology. Establishing a psychological community at a Brazilian university. Revista Interamericana de Psicología, 46(1), 135-142.

Falcone, R. (2012). Psicología en Argentina: impronta europea y carácter nacional. Revista Interamericana de Psicología, 46(1), 87-98. doi: 10.30849/rip/ijp.v46i1.184

Falcone, R. (2015). Las relaciones sociales de género en la comunidad psicoanalítica argentina (1942-1969). Revista Científica de UCES, 9(1), 90-121.

Ferrari, J. F. (2013). Virgilio Ducceschi y el primer laboratorio de psicología experimental en Córdoba, Argentina (1907). Estudos e Pesquisas em Psicologia, 13(2), 779-805. Recuperado de https: //www.e-

publicacoes.uerj.br/index.php/revispsi/article/view/8437/6273

Ferrero, C., Iemme Khoury, V., Todisco, R., \& Scherman, P. (2016). Inicios de la investigación experimental en psicología en Córdoba en el período 1960-1990. Revista Argentina de Ciencias del Comportamiento, 8(2), 58-66. Recuperado de https: // revistas.unc.edu.ar/index. php/racc/article/view/10023

Flores-Mendoza, C., Ardila, R., Gallegos, M., Sampaio Braga, L., Miranda Carvalhais S. B., \& Andrade, M. (2016). Hans Eysenck 
in Latin America: His influence in the psychology, the study of personality and individual differences. Personality and Individual Differences, (103), 68-73.

French, J. L. (1988). Grandmothers I wish I knew: contributions of women to the history of school psychology. Professional SchoolPsychology, 3(1), 51-68.

Fogliatto, H. M. (1963). Individual differences in thought processes (Tesis de Doctorado). Loyola University, Chicago. Retirado de https://ecommons.luc.edu/luc diss/785/

Fogliatto, H. M. (1999). El paradigma informático en orientación vocacional. Orientación y Sociedad, 1, 1-11.

Furumoto, L. (1992). Joining separate spheres: Christine LaddFranklin, womanscientist (1847-1930). American Psychologist, 47(2), 175-182.

Furumoto, L., \& Scarborough, E (1986). Placing women in the history of psychology: The first American women psychologists. American Psychologist, 4(1), 35-42.

Giménez, M. C. (2007). Mujeres en la historia de la psicología. Revista de Historia de la Psicología, 28(2/3), 281-290.

Insúa, A. M. (1970). Some personality variables in relation to problema-solving processes of college students (Tesis de doctorado). Loyola University, Chicago. Retirado de https: //ecommons.luc.edu/luc_diss/1027/

Insúa, A. M. (1975). Parapsicología, ¿ficción o realidad? Soviet Psychology. Psicología Médica, 1, 127-128.

Insúa, A. M. (1981). An aesthetic value scale of the Rorschach. Journal of Clinical Psychology, 37(1), 189-192. doi: 10.1002/1097-4679(198101)37: 1<189: : AIDJ CLP2270370139>3.0.CO;2-D

Insúa, A. M., \& Loza, S. M. (1986). Psychometric Patterns on the Rorschach of Healthy Elderly Persons and Patients with Suspected Dementia. Perceptual and Motor Skills, 63(2), 931936. doi: 10.2466/pms.1986.63.2.931

Insúa, A. M., \& Loza, S. M. (1988). Evaluación de los signos de organicidad, según Piotrowsky, en el Rorschach de pacientes con un comienzo de demencia. Acta Psiquiátrica y Psicológica de América Latina, 34(3), 243-250.

Insúa, A. M., Rimoldi, H. J. A., \& Erdman, J. B. (1972). Análisis factorial del test de Holtzman. Boletín del CIIPME, (12).

Insúa, J. A. (2013, Enero 15). Foto entrevista al Dr. Jorge A. Insúa [Archivo de vídeo]. Recuperado de https: //www.youtube.com/watch?v=BQex0G5206g

Jacó-Vilela, A. M., Degani-Carneiro, F., \& Novaes Messias, M. C. (2012). Mujer y psicología: la presencia femenina en la historia de la psicología de Río de Janeiro. Investigaciones en Psicología, 17(2), 11-12. 
Klappenbach, H. (2001) Dos editoriales en los comienzos de la profesionalización de la psicología en Argentina. Memorandum, 1, 61-71. Recuperado de http://www. fafich.ufmg.br/ memorandum/artigos01/klappenba ch01.htm

Klappenbach, H. (2006). Recepción de la Psicología Alemana y Francesa en la Temprana Psicología Argentina. Mnemosine, 2(1), 75-86.

Klappenbach, H. (2014). Acerca de la metodología de la investigación en la historia de la psicología. Psykhé, 23(1), 1-12. doi: 10.7764 /psykhe.23.1.584

Klappenbach, H. (2018). Cambios en los primeros perfiles de formación en el campo de la Psicología Argentina. Desde la Planificación Estatal a una Profesión Liberal. Revista de Historia de la Psicología, 39(3), 18-27. Recuperado de https: //www.revistahistoriapsicologia.es/revista/2018-vol-39n\% C3\% BAm-3/

Lev, M., Rimoldi, H. J. A., Paiva, R., \& Arcilla, R. A. (1969). The quantitative anatomy of simple complete transposition. The American J ournal of cardiology, 23(3), 409-416.

López Miranda, R., Rota Júnior, C., Baker, D., \& Dias Cirino, S. (2016). The Bello Horizonte Teacher's College Laboratory. Circulating Psychology in Brasil. Revista Mexicana de Análisis de la Conducta, 42(2), 179-199. doi: 10.5514/rmac.v42.i2.57026

Miguez, G., Betancourt Mainhard, R., \& Villarroel, P. V. (2010). Historia de los laboratorios de psicología en Chile. In M. A. Laborda \& V. E. Quezada (Eds.), Notas históricas de la psicología en Chile. Santiago: Editorial Universitaria.

Milar, K. S. (2000). The first generation of women psychologists and the psychology of women. American Psychologist, 55(6), 616619.

Montero, I., \& León, O. G. (2005). Sistema de clasificación del método en los informes de investigación en psicología. International Journal of Clinical and Health Psychology, 5(1), 115-127. Recuperado de https: //www.redalyc. org/html/337/33701007/

Ostrovsky, A. E. (2008). La Sociedad de Psicología en Argentina (1908-1913): Treinta y nueve hombres y una mujer. Revista de Historia de la Psicología, 29(2), 55-67. Recuperado de https://www.revistahistoriapsicologia.es/revista/2008-vol-29n\% C3\% BAm-2/

Ostrovsky, A. E. (2011). Mujeres en los Archivos de Pedagogía y Ciencias Afines: colegas, alumnas e hijas. III Congreso Internacional de Investigación y Práctica Profesional en Psicología XVIII Jornadas de Investigación Séptimo Encuentro de Investigadores en Psicología del MERCOSUR. Facultad de 
Psicología - Universidad de Buenos Aires, Buenos Aires. Recuperado de https: //www.aacademica.org/000-052/143

Ostrovsky, A. E. (2014). La historia de la psicología en clave crítica: Juicios y prejuicios en el análisis de la psicología de la "mujer universitaria" en Argentina a comienzos del siglo XX. Quaderns de Psicologia, 16(1), 201-212.

Ostrovsky, A. E., \& Moya, L. A. (2013). Participación de mujeres en dos momentos de la psicología argentina. $\mathrm{V}$ Congreso Internacional de Investigación y Práctica Profesional en Psicología, XX Jornadas de Investigación, IX Encuentro de Investigadores en Psicología del Mercosur, Facultad de Psicología de la Universidad de Buenos Aires. Recuperado de http: //www.aacademica.org/000-054/134

Paiva, R. E. (1968). Tempo and problem-solving processes (Tesis de Doctorado). Loyola University, Chicago. Recuperado de https: //ecommons. luc.edu/luc_diss/1001/

Pérez, E., \& Passera, J. (2004). Hermelinda Fogliatto, pionera de la orientación profesional en la Argentina (4/7/1925-9/10/03). Orientación y Sociedad, 4, 1-2.

Piñeda, M. A. (2005). El Padre Leonardo Castellani y la Psicología Argentina. Revista de Historia de la Psicología, 26(1), 67-100.

Piñeda, M. A. (2007). La creación de la carrera de psicología en universidades católicas argentinas. Memorandum, 12, 6-29.

Piñeda, M. A. (2012). Psychology Publications by professors at Argentine Psychology Programs: 1958-1982. Interamerican Journal of Psychology, 46(1), 111-122.

Piñeda, M. A. (2014). Psicología experimental francesa y cultura científica en los inicios de la carrera de psicología de la Universidad Nacional de Cuyo (San Luis, Argentina). Psicologia em Pesquisa, 8(1), 66-76. doi: 10.5327/Z19821247201400010007

Piñeda, M. A. (n.d.). Eva Borkowska de Mikusinski, H. J. Eysenck y los estudios científicos de la personalidad en Argentina. Estudos e Pesquisas em Psicologia. Sin publicar.

Piñeda, M. A., \& Scherman, P. (2016a). Estudios experimentales de la percepción desde la profesionalización de la psicología en Argentina. Estudos e Pesquisas em Psicologia, 16(1), 295-316.

Piñeda, M. A., \& Scherman, P. (2016b). S. S. Stevens, M. Guirao y los estudios psicofísicos en Argentina. Revista Mexicana de Análisis de la Conducta, 42(2), 153-178.

Prado da Fonseca, L. E., Rocha Silva da Rosa, H. L., \& Arruda Leal Ferreira, A. (2016). Yes, nosotros tenemos a Wundt: Radecki y la historia de la psicología en Brasil. Tesis Psicológica, 11(1), 36-53.

de https: // www.redalyc.org/pdf/1390/139050020004.pdf 
Radtke, H. L., Hunter, M., \& Stam, H. J. (2000). In memoriam as in life: gender and psychology in the obituaries of eminent psychologists. Canadian Psychology, 41(4), 213-229.

Rodríguez Sturla, P. (2001a). Carolina Tobar García: La infancia con dificultades educativas. In L. Rossi (Ed.), Psicología, su inscripción universitaria como profesión, una historia de discursos y prácticas. Buenos Aires: Eudeba.

Rodríguez Sturla, P. (2001b). Telma Reca: La institucionalización de la atención infantil. In L. Rossi (Ed.), Psicología, su inscripción universitaria como profesión, una historia de discursos y prácticas. Buenos Aires: Eudeba.

Rimoldi, H. J. A. (1995). Testimonio autobiográfico. Cuadernos Argentinos de Historia de la Psicología, 1(1/2), 275-300.

Rimoldi, H. J. A., Haley J. V., \& Fogliatto, H. M. (1962). Training in problem-solving. Chicago: Loyola University, Loyola Psychometric Laboratory.

Rimoldi, H. J. A., Fogliatto, H. M., Haley, J. V., Reyes, I., Erdmann, J.V., \& Zacharia, R. (1964). The test of diagnostic skills. Chicago: Loyola University, Loyola Psychometric Laboratory.

Rimoldi, H. J. A., \& Insúa, A. M. (1976). Invariabilidad de la estructura factorial del "California Personalityl nventory" (CPI) Revista Latinoamericana de Psicología, 8(3), 399-409.

Rimoldi, J. H. Insúa, A. M., \& Erdman J. B. (1975). Personality dimensions as assessed by projective and verbal instruments. J ournal of Clinical Psychology, 31, 529-539.

Sacchi, J. C. (1963). Applicazione di una Tecnica di Gruppo nel Trattamento di AlcoolistiCronici (Tesi di Perfezionamento). UniversitàCattolica del Sacro Cuore, Milano.

Sacchi, J. C. (1977). Variables de ansiedad y hostilidad en el test de Holtzman. Revista Latinoamericana de Psicología, 9(2), 271276.

Salas Contreras, G. (2012). La influencia europea en los inicios de la historia de la psicología en Chile. Revista Interamericana de Psicología, 46(1), 99-110.

Seglares Claretianos (2005). Vida de los grupos y comunidades: testimonio de dos hermanas de la región Argentina-Uruguay. Consejo General del Movimiento Seglar Claretiano, (92).

Sprung, H., \& Sprung, L. (1996). Women in Psychological History. Forms of integration in Psychology and Women's lecturing activities at German Psychology Congresses: 1904-1978. Revista de Historia de la Psicología, 17(1/2), 185-202.

Universidad Nacional de Cuyo (n.d.). Libro de egresados. Sin publicar.

Winkler, M. I. (2004). Otra historia de la psicología: una tríada de mujeres. Revista Interamericana de Psicología, 38(1), 5-14. Recuperado

de 
http://psicorip. presser.net. br/Resumos/PerP/RIP/RI P036a0/RIP 03802.pdf

Winkler, M. I. (2007). Pioneras sin monumentos: mujeres en Psicología. Santiago: Lom.

Winkler, M. I. (2015). Historias de mujeres en la psicología chilena: contribuciones de Lola Hoffmann, Héliètte Saint Jean y Vera Kardonsky. Psykhe, 24(1), 1-11. doi: 10.7764/psykhe.24.1.658

Winkler, M. I., \& Reyes Espejo, M. I. (2014). Mujeres, psicólogas y psicoanalistas en Chile y Argentina: historias que marcaron diferencias. In G. Salas (Ed.), Historias de la Psicología en América del sur: Diálogos y perspectivas. La Serena: Nueva Mirada.

\section{Endereço para correspondência \\ María Andrea Piñeda}

Universidad Nacional de San Luis

Facultad de Psicología - Consejo Nacional de Investigaciones Científicas y Técnicas Ejército de los Andes, 950, Edificio Plácido Horas, $1^{\circ}$ piso, oficina 59, CP 5700, San Luis, Argentina

Endereço eletrônico: mapineda@unsl.edu.ar

Recebido em: 19/12/2018

Reformulado em: 29/01/2019

Aceito em: 30/01/2019

\section{Notas}

* La autora es Doctora en Psicología; Investigadora Adjunta del Consejo Nacional de Investigaciones Científicas y Técnicas y Profesora Adjunta de la Facultad de Psicología de la Universidad Nacional de San Luis donde dirige el Museo de Historia de la Psicología.

Esta investigación ha sido realizada con el apoyo y financiamiento del Consejo Nacional de Investigaciones Científicas y Técnicas y de la Universidad Nacional de San Luis.

Se agradece a la Dra. Ana Elisa Ostrovsky por sus aportes y sugerencias.

Este artigo de revista Estudos e Pesquisas em Psicologia é licenciado sob uma Licença Creative Commons Atribuição-Não Comercial 3.0 Não Adaptada. 